\title{
A multi-channel gel electrophoresis and continuous fraction collection apparatus for high throughput protein separation and characterization
}

\author{
Megan Choi ${ }^{1}$, Robert A. Nordmeyer ${ }^{2}$, Earl Cornell ${ }^{2}$,Ming Dong ${ }^{1}$, Mark D. Biggin ${ }^{1}$, \\ and Jian Jin $*^{2}$ \\ ${ }^{1}$ Genomics Division, Lawrence Berkeley National Laboratory, CA; ${ }^{2}$ Engineering \\ Division, Lawrence Berkeley National Laboratory, CA \\ *Corresponding Author: \\ Jian Jin \\ 1Cyclotron Road, MS 46-125 \\ Lawrence Berkeley National Laboratory \\ Berkeley, CA 94720 \\ Tel: (510) 486-4604 \\ Fax: (510) 486-4633 \\ Email: JJin@1bl.gov
}

\begin{abstract}
To facilitate a direct interface between protein separation by polyacrylamide gel electrophoresis (PAGE) and protein identification by mass spectrometry, we developed a multi-channel system that continuously collects fractions as protein bands migrate off the bottom of gel electrophoresis columns. The device was constructed using several short linear gel columns, each of a different percent acrylamide, to achieve a separation power similar to that of a long gradient gel. A "Counter Free-Flow" elution technique then allows continuous and simultaneous fraction collection from multiple channels at low cost. We demonstrate that rapid, high resolution separation of a complex protein mixture can be achieved on this system using SDS-PAGE. In a 2.5-hour electrophoresis run, for example, each sample was separated and eluted into 48 to 96 fractions over a mass range of $\sim 10$ to $150 \mathrm{kD}$; sample recovery rates were $50 \%$ or higher; each channel was loaded with up to $0.3 \mathrm{mg}$ of protein in $0.4 \mathrm{ml}$; and a purified band was eluted in 2-3 fractions (200 $\mu 1 /$ fraction). Similar results were obtained when running native gel electrophoresis, but protein aggregation limited the loading capacity to about $50 \mu \mathrm{g}$ per channel and reduced resolution.
\end{abstract}

KEYWORDS: continuous elution electrophoresis/counter free-flow/D. vulgaris /high throughput/multi-channel electrophoresis.

\section{$1 \quad$ Introduction}

Most proteomic analyses combine a multi-dimensional separation scheme with protein identification by mass spectrometry. This reflects the simple fact that complex mixture of proteins must be well separated and simplified prior to identification, and only mass 
spectrometry has the adequate sensitivity and throughput for subsequent analysis. Polyacrylamide gel electrophoresis (PAGE) [1-3] is one of the most effective protein separation techniques and has been incorporated into various multi-dimensional schemes. For example, traditional two-dimensional gel electrophoresis (2DE) is well established as a robust method for separating complex protein lysates obtained from cells. The power of 2DE stems from the fact that both isoelectrical-focusing (IEF) $[4,5]$ and SDS gel electrophoresis are highly resolving, orthogonal techniques. For membrane proteins, twodimensional blue native/SDS gel electrophoresis (2D BN/SDS-PAGE) [6] has been the method of choice for intact proteins and protein complexes. Similarly, PAGE is an important step for further separating polypeptides after "affinity tagged" purification [79]. However, a major limitation of all these forms of PAGE, compared with many liquid chromatography techniques, is that they are not readily interfaced with mass spectrometry because it is inconvenient to extract separated protein bands from the gel for further processing. To harvest separated proteins, one must either physically cut them out as gel slices or elute them from the bottom of gels. Large scale "gel-cutting" has been used effectively in proteomics and protein interaction projects [7-11], but it is generally labor intensive. Gel-cutting robots-for instance, the "Ettan Spot Picker" from GE Healthcare's Life Sciences (Piscataway, NJ)—exist, but their accessibility is limited. To elute proteins from the bottom of gels, there are at least two devices on the market: the "Model 491 Prep Cell" and "Whole Gel Eluter" from Bio-Rad (Hercules, CA). However, these are not widely used, partially because they can process only one or two samples at a time and because sample loss and dilution are significant.

As part of the US Department of Energy's Genomics GTL program, the Protein Complex Analysis Project (PCAP) (http://vimss.lbl.gov/projects/pcap.html) is developing high throughput methods and pipelines to rapidly purify and identify the majority of stable protein complexes in a given microbe. Like strategies used previously to identify protein complexes, some of the high throughput pipelines we are developing as part of PCAP use SDS or native PAGE for protein separation and purification, followed by protein identification by mass spectrometry $[7-9,12]$. To meet the GTL program's needs, it will be necessary to develop methods that can separate and elute thousands of protein samples by gel electrophoresis as rapidly as possible.

To increase throughput, here we present an alternative, easy-to-use multi-channel electrophoresis system that separates complex mixtures of proteins while automatically capturing separated bands into a liquid fraction collector over a broad mass/size range. We have devloped a scheme that: (1) uses a combination of two or three short (2-3-cm long) linear gel columns of different polyacrylamide concentrations to achieve a separation power similar to a typical 10-15-cm long gradient gel; and (2) enables continuous fraction collection from multiple gel columns. Figure 1 illustrates this scheme. Two $\sim 2 \mathrm{~cm}$ long linear native 5 and $8 \%$ polyacrylamide gels are set in glass tubes and the resulting gel columns are stacked one on top of the other to mimic a 5-8\% gradient gel. After an initial (typically, around 30 minutes) electrophoresis run, the top gel column is moved and placed on another elution unit (unit 2 in Fig. 1).

Electrophoresis is then continued on both units to further separate protein bands. The 
proteins that as a result elute from the bottom of each gel column are collected in parrallel on fraction collectors (Fig. 1, bottom).

For this scheme to be successful, it was critical to find an effective way to collect protein bands as they elute and to allow simultaneous operation of multiple channels at a low cost. Important issues that needed to be resolved in fraction collection included preventing interruption of electrophoresis, maintaining the continuity of collection, and reducing sample loss, resolution loss, and dilution of protein bands. Several approaches, although primarily designed for capillary electrophoresis (CE), have been previously investigated by others [13-15]. Of these, three are of particular interest: (1) Sweep Liquid, in which liquid is swept through a standard liquid chromatographic detector, resulting in continuous collection and uninterrupted electrophoresis but high sample dilution [13]; (2) On-line Frit, in which a frit structure is attached to the outlet end of the capillary to isolate electrical conductivity from the elution buffer flow [14]; and (3) Coaxial Sheath Flow: in which a sheath flow is built around the capillary to confine the sample flow and provide the electrical connection to the electrode [15]. Each of these methods has its advantages and disadvantages, but none is ideal for our needs. We therefore designed and constructed an elution device employing a different "Counter Free-Flow" approach. It is similar to the Coaxial Sheath Flow method in that it uses electrophoresis buffer as the media to establish electrical connection between the gel column and the anode, but our method also employs a bulk flow of buffer solution to drain separated bands migrating off the gel column. Our "Counter Free-Flow" technique also enables simple scale-up to multi-channel operation at a low cost since it does not use expensive pumps to control the flow rate.

\section{$2 \quad$ Materials and methods}

\subsection{Capturing protein bands}

Our "Counter Free-Flow" technique uses a machined conduit of acrylic glass and a fused-silica capillary tube. As shown in Figure 2, the conduit provides the interface between electrophoresis and the collection of eluted biomolecules. The gel column can be easily inserted into the upper cup of the conduit and the taper (a funnel) at the bottom of the cup provides physical support to the gel. This arrangement reduces the diameter of eluting bands from $7 \mathrm{~mm}$ to $3 \mathrm{~mm}$ over a vertical distance of $2 \mathrm{~mm}$ as they move down the taper. Below the taper is the final portion of the conduit, a straight tube that is $12-\mathrm{mm}$ long, with a 4.5-mm outer diameter (od); in the middle of the length of this tube, four 1.0 $\mathrm{mm}$ diameter holes are drilled perpendicular to the central axis of the tube. These holes allow electrical currents to flow between the gel column and the anode and running buffer to flow in. The inner diameter (id) of the straight tube is $3 \mathrm{~mm}$ above the holes and only $1.53 \mathrm{~mm}$ below the holes. The increased electrical field within the straight tube provides additional acceleration to eluting biomolecules once they enter the gel-free buffer solution. A narrow-bore glass capillary tube with a sleeve (a $50 \mathrm{~mm}$ long PEEK tube, $0.5 \mathrm{~mm}$ id and $1 / 16^{\text {th }}$ inch od) is inserted into the bottom of the conduit. The PEEK tube ends about $1 \mathrm{~mm}$ below the four holes and the capillary tube $2 \mathrm{~mm}$ below the taper. When charged molecules reach the tip of the capillary tube they are subjected to an inward drag force generated by the counter-flow of buffer solution. As the rate of the counter-flow increases, it overcomes the electrical force and sweeps the biomolecules 
down into the capillary tube and deposits them into a fraction collector below. The buffer flow can be gravity-driven, and thus does not require expensive pumps. The buffer flow is controlled by adjusting the length and inner diameter of the capillary tube. The relative vertical position of the capillary tube within the straight tube is an important parameter that affects the capture efficiency of eluted biomolecules (see below for further details). Using this approach, many channels can be operated simultaneously.

\subsection{Apparatus}

Based on the scheme described above, a prototype, 16-channel instrument (see Fig. 3) has been constructed and tested. We used gel boxes that each house four short $(3 \mathrm{~cm}$ long) glass tubes for forming the lower and middle gel segments in the "multi" mode (described below), and boxes that house four longer $(5 \mathrm{~cm}$ or $12 \mathrm{~cm})$ glass tubes for either the upper most gel segment in the multi mode or the only gel column in the "single" mode (described below) (Fig. 3). Each box includes an upper run buffer container, which includes a Platinum electrode for use as a cathode when needed, and the four glass tubes, spaced $18 \mathrm{~mm}$ center-to-center (Fig. 1 shows a simpler version with just one tube per box). The bottom plate of the buffer container in each box has four precisely machined (clearance) holes. The inner diameter of the holes (at their base) tightly matches the outer diameter of the glass tubes. When the glass tubes are inserted from the bottom and glued, the tight fit ensures the tubes will be straight and evenly spaced. For the lower (short) gel boxes, the top of each tube ends about $5 \mathrm{~mm}$ below the top of the hole (Fig. 1). Above this, the inner diameter of the holes is slightly larger and there is a shallow taper at the top of the hole. This structure makes it easy to insert additional gel boxes from above and provides a good seal at the interface. For the upper gel boxes, the glass tube protrudes $2 \mathrm{~cm}$ above the hole, providing a large length of tube above the top of where the gel will be set for loading samples. Gels are polymerized in the glass tubes over night. Prior to stacking multiple gel boxes together, the space above each gel (in the hole on top of the lower gel boxes) is filled with run buffer to prevent air bubbles from being trapped between the gel columns. The upper gel box is also filled with run buffer, leakage being prevented at the "ducking" interface because the cross-linked polymer gels seal the glass tubes. Protein leakage is also prevented since the interface is electrically floated, therefore, the electrical field remains confined within the tube and there is no other force to drive protein radialy. After the initial electrophoresis run and subsequent un-stacking of the gel boxes, the gaps above the lower gel columns are filled with run buffer again and the electric field is turned on for 30-60 seconds to drive protein molecules remaining in solution into the lower gel column. Finally, the buffer containers for each separated box are filled up and electrophoresis is continued.

The elution unit, which also serves as the lower buffer container, is formed by attaching a base plate holding four capillary tubes $(320 \mu \mathrm{m} \mathrm{id}, 450 \mu \mathrm{m}$ od and $15-25 \mathrm{~cm}$ long [part \# TSP320450, Polymicro Technologies, Phoenix, AZ]) and an O-ring gasket to a buffer container body, which includes a Pt electrode (anode) and a buffer inlet and outlet (Fig. 2 shows an example for a single gel elution unit). At the top of the elution unit, there are four precisely machined holes in which plastic conduits are inserted that receive the gel column from the segment above. A buffer inlet is attached to the bottom of the base plate so that fresh buffer is supplied to the capillaries directly. The outlet is attached to a side wall at a height that defines the level of buffer in the chamber during 
electrophoresis, which is about $5 \mathrm{~mm}$ above the taper in the conduit to ensure that the bottoms of the gel columns are submerged in buffer (Fig. 2). The buffer level is maintained either by connecting the inlet to a large (4-L) glass buffer container sitting on a lab jack that is set slightly higher than the elution unit or by using a small, inexpensive dual-channel peristaltic pump. The excess flow not consumed by the four capillaries is drained through the outlet and disposed. There is also a pinch-valve on the outlet line, which is closed to raise the buffer level to cover the tops of the conduits only when the gel columns are being inserted from above.

The glass capillary tubes in the elution unit base plate are attached to a holder, under which a motorized fraction collector using standard 96-well plates is located. There are two identical but individually addressable fraction collectors, each of which supports two 4-channel electrophoresis units.

\subsection{Modes of operation}

The instrument can be operated in two different modes. In the "single" mode, the sample is loaded above a single-piece, long gel column, and the proteins are separated and eluted directly into the fraction collector. Since there is only one segment of gel used in this mode, it can achieve effective separation over a finite mass range only, the range being chiefly determined by the concentration of polyacrylamide used. In the "multi" mode, two or three segments of gel columns, each with a different gel concentration, are stacked on top of one another during the initial electrophoresis run, the gels of lower concentration being placed above those of higher concentration. After the faster migrating protein bands have entered the lower gel segment, the upper segments are removed and put onto other elution units where the electrophoresis of slower migrating proteins is completed and the separated bands eluted and collected. The single mode is fast and effective if the application targets a specific band. The single mode can also be used where multiple gel columns of different percent acrylamide target a broader band range of polypeptides in cases where sample consumption is less of an issue. The multi mode is more efficient in separation and sample use but needs more control and parameter optimization in operation. For example, the condition and time of when to separate the two stacked gel columns must be empirically determined. The multi mode set-up functions similarly to that of a traditional analytical mode for protein separation.

\subsection{SDS and native PAGE gels}

To cast either SDS or native PAGE gels, first the bottom of each gel column was sealed using thin plastic film, then gel solution was poured into the columns to the desired length and a 5-mm deep layer of water-saturated butanol was added on top to separate the gel solution from air and to maintain a flat gel surface. The gel solution was made fresh each time using stock solutions of 30\% acrylamide (Bio-Rad, Cat. \#161-0156), $375 \mathrm{mM}$ Tris $\mathrm{HCl}$ buffer ( $\mathrm{pH} 7.8), 0.1 \%(\mathrm{v} / \mathrm{v})$ TEMED and $0.03 \%(\mathrm{w} / \mathrm{v})$ ammonium persulphate. For native gels only, once the upper most separation gel was set and the butanol removed, a $4 \%$ stacking gel was poured on top using $125 \mathrm{mM}$ Tris $\mathrm{HCl}(\mathrm{pH} \mathrm{6.8)}$ ) as the buffer, and butanol was added again. The stacking gel length was kept at least twice the depth of the sample to be loaded, with the minimum length being $1 \mathrm{~cm}$. Native gels were run in $1 \mathrm{X}$ running buffer (25mM Tris, $191.8 \mathrm{mM}$ glycine) and SDS gels were run in the same buffer with $0.2 \%(w / v)$ SDS. For the multi mode, several gel columns were stacked after 
removal of the butanol, with a small quantity of running buffer being left to fill the short (1-2 mm) gap between the gel segments. Typical electrophoresis conditions were 20-30 volts/cm, with a power limit of 1-2 watts/column.

\subsection{Protein samples for electrophoresis}

Crude Desulfovibrio vulgaris (D. vulgaris ) extracts ( $\sim 5 \mathrm{mg} / \mathrm{ml}$ protein) were prepared for electrophoresis by addition of $1 / 3$ volume of $6 \mathrm{X}$ sample loading buffer (150mM Tris, $1.15 \mathrm{M}$ glycine, $60 \%$ (v/v) glycerol, $0.03 \%$ (w/v) bromophenol blue). For samples for SDS PAGE, the loading buffer contained an additional $18 \%(\mathrm{v} / \mathrm{v})$ of SDS and samples were denatured at $95{ }^{\circ} \mathrm{C}$ for 10 minutes prior to loading. For SDS and native gel electrophoresis, sample loading was typically $0.4 \mathrm{ml}$ (containing $0.32 \mathrm{mg}$ protein) per channel. For more purified samples that contain high salt, such as the fractions from a hydrophobic interaction column (HIC) used in Figure 6, a desalting column from GE HealthCare (PD-10 Column, Cat. \#17-0851-01) was used to remove salt and exchange the samples into $125 \mathrm{mM}$ Tris $\mathrm{HCl}$ buffer ( $\mathrm{pH}$ 6.8). These samples were then concentrated using a Millipore 3K Amicon Ultra column (UFC800324) to a volume of 200-600 $\mu \mathrm{l}$ per fraction. HIC samples were prepared for native electrophoresis by adding $1 / 3$ volume of $6 \mathrm{X}$ stacking gel sample loading buffer (187.5 mM Tris $\mathrm{HCl}$ [pH 6.8], 60\% (v/v) glycerol, $0.036 \%(\mathrm{w} / \mathrm{v})$ bromophenol blue). A typical loading of an HIC sample was $135 \mu \mathrm{g}$ in $0.2 \mathrm{ml}$ volume per channel.

\subsection{Slab gel analysis of eluted fractions}

To monitor and evaluate the separation and elution results of our free-flow electrophoresis instrument, fractions were sampled and analyzed using more traditional slab gels. A $12.5-\mu 1$ portion from each eluted fraction sampled was mixed with $3 \mu 1$ of either 6X sample loading buffer (150mM Tris, $1.15 \mathrm{M}$ glycine, $60 \%$ glycerol (v/v), $0.03 \%$ (w/v) bromophenol blue), for native gels, or the same buffer including 18\% SDS, for SDS-PAGE. These mixtures were then loaded onto slab gels: Bio-Rad's Criterion Tris$\mathrm{HCl} \mathrm{Gel,} \mathrm{4-15 \%} \mathrm{(Cat.} \mathrm{\# 345-0029)} \mathrm{for} \mathrm{native} \mathrm{samples} \mathrm{or} \mathrm{the} \mathrm{Criterion} \mathrm{4-20 \%} \mathrm{(Cat.} \mathrm{\# 345-}$ 0034 ) for SDS samples. Native gels were run at $200 \mathrm{~V}$ in $1 \mathrm{X}$ gel running buffer $(25 \mathrm{mM}$ Tris, $191.8 \mathrm{mM}$ glycine), and SDS gels at $200 \mathrm{~V}$ in $1 \mathrm{X}$ gel running buffer with $0.2 \%$ SDS. Gels were run until the dye front reached the bottom of the gel. Gels were stained using Invitrogen's Silver Quest staining kit (LC6070).

\section{$3 \quad$ Results and discussion}

\subsection{Optimizing the device}

Multiple geometrical and dynamic parameters can affect the collection efficiency and resolution of this device, as suggested by the description above in combination with Figure 2. Through a series of trial and error modifications and prototype versions, we found that the most critical of these parameters were the electrophoresis electrical field, flow rate of the elution buffer, and relative vertical location of the capillary tube along the straight tube at the bottom of the conduit. Given the structure and geometry of the current collection device, the optimal electrical field was $20-30$ volts/cm, flow rate was $\sim 125$ $\mu 1 /$ minute, and the position of the capillary was $2 \mathrm{~mm}$ below the tapered section of the conduit. Using these conditions, approximately $50 \%$ of the protein loaded could be 
recovered in the eluted fractions, with typically $0.2-0.5 \mathrm{ml}$ of loaded sample being eluted in fractions totaling $0.2-0.6 \mathrm{ml}$.

These conditions were empirically determined. The maximum field that could be applied for electrophoresis was 30 volts $/ \mathrm{cm}$. Going beyond this threshold resulted in local over-heating, causing gas bubbles to form between the bottom of the gel column and the taper, which interrupts electrophoresis. The balance between recovery efficiency and dilution of eluted bands is determined by the counter buffer flow rate. This flow was optimized by monitoring the collection of a band of loading dye. When the flow rate was low, some dye molecules would go around and bypass the tip of capillary tube, and ultimately leak out from the conducting holes. As the flow rate increased, the leak diminished to the point of being undetectable. (see Supplementary Fig. 1). We defined the optimal flow as the minimum rate at which there is no detectable leak of dye because proteins are larger and less mobile than dye molecules. If we capture most dye molecules, we should have also captured most proteins and have prevented unnecessary dilution.

\subsection{Elution of SDS-PAGE gels}

To demonstrate that this instrument works, we first used it to separate and elute a mixture of denatured proteins by SDS gel electrophoresis. Figure 4 gives an example of a set of defined marker protein bands collected by this instrument. After an initial 32-min run, 6 bands of pre-stained protein standards (Bio-Rad Co.) of $50 \mathrm{kD}$ or smaller entered the lower, second gel segment (6.5\%), while the other 4 bands of $75 \mathrm{kD}$ and higher remained in the upper, first gel segment (4.5\%). We then removed the top gel segment to another elution device and completed electrophoresis and elution on both units. The concentration of dye in the collected fractions suggested the approximate correspondence between the pattern of elution and the bands in gels, and this was confirmed by subsequently running these fractions on an analytical SDS slab gel (data not shown).

A second test was then performed using a complex mixture of proteins from a crude extract of $D$. vulgaris. In Figure 5 ( $\mathrm{a}, \mathrm{b}$ and $\mathrm{c}$ ) the single mode was used for separation and identical samples were loaded onto 7\%, 10\% and 12\% linear SDS gels. In Figure $5(\mathrm{~d}$, e and f) two-staged $(7 \%+10 \%, 8 \%+12 \%$, and $10 \%+12 \%)$ gels in the multi mode were employed. As shown by a slab-gel analysis of the fractions eluted, a common feature displayed in all of these gels is that each fraction contains only a narrow band of proteins of similar molecular weights with only a modest overlap in the proteins contained in neighboring fractions, illustrating that this instrument works as designed. Two additional obvious features are: (1) the very smallest molecular weight proteins are not well resolved, with many bands eluted into a single fraction; and (2) larger molecular weight polypeptides migrate too slowly, such that a single band is "stretched" over many fractions or may not elute at all within a reasonable time frame. The data suggest that for high speed, high resolution separation, a $2-\mathrm{cm}$ long $12 \%$ gel works well for proteins up to $25 \mathrm{kD}$, a $10 \%$ gel for $25-100 \mathrm{kD}$, and 7 or $8 \%$ gel for $100 \mathrm{kD}$ and above. To cover a broader range, it is necessary to use the multi mode. Excellent results are obtained by a two-staged $10 \%$ and $12 \%$ gel for proteins of $10-100 \mathrm{kD}$ (Fig. 5 (f)); a combination of $8 \%$ and $12 \%$ can extend that range to $150 \mathrm{kD}$ (Fig. 5 (e)). The $7 \%+10 \%$ combination in 
Figure 5 (d) shifted the coverage to $25-150 \mathrm{kD}$. A three-staged gel system might be needed if one wants to cover the entire range for denatured proteins (from a few $\mathrm{kD}$ to approximately $200 \mathrm{kD}$ ). The capacity of this system was relatively high, with good results being obtained when up to $500 \mu \mathrm{g}$ of protein were loaded per gel column.

We have found that the protein separation reproducibility of this system is similar to other PAGE instruments, based on approximately 100 test runs performed over 2 years. In each run, the system was disassembled and reassembled with new buffer and gels, and multiple samples (identical replicates or different) were loaded and fractionated. For the same running conditions, the dye front arrival times, from lane-to-lane and dayto-day, typically varied by only 1 to 2 minutes (see Supplementary Fig. 1) and the position shift of corresponding protein bands eluted and collected was only plus or minus one fraction (supplementary Fig. 2).

\subsection{Elution of native gels}

To understand the separation characteristics of linear, native polyacrylamide gels in our free flow electrophoresis system, we first developed protocols for casting and operating different percent gels in the single mode. Figure 6 shows typical results using 2-cm long gels. The $4 \%$ gel provided good separation for protein complexes of masses $400 \mathrm{kD}$ and above, the $6 \%$ gel for $150-350 \mathrm{kD}$, and the $8 \%$ gel for $45-180 \mathrm{kD}$. However, we also noticed that native gels suffer from what appears to be a protein aggregation problem that reduces their effectiveness, even when the amount of protein loaded is limited to $50 \mu \mathrm{g}$. Using a sample that contained only a few large molecular weight protein complexes, a smear of apparently large molecular weight proteins are eluted that do not correspond to any input proteins and many of them are larger than any input protein (see Fig. 7). SDS slab gel analysis of the same eluted protein fractions showed that these "novel" large proteins mostly consisted of small molecular weight polypeptides found in the input sample, and thus appear to be aggregates created during native electrophoresis in the free flow device.

We also tested a two stage combination of 2-cm long gels of $5 \%$ and $8 \%$ in the multi mode. Figure 8 illustrates the results. The range of fractionation seen is consistent with that in the single mode gels (see Fig. 7 (a)). However, over much of the size range, the resolution of the native free-flow gels is lower than that of the SDS free flow gels (compare Fig. 8 with Fig. 5). Due to the difficulty of correctly judging when to separate the two stages for elution, there is also an apparent preferential loss of proteins between 140 and $-400 \mathrm{kD}$. For these reasons, native counter free-flow electrophoresis appears less effective than SDS counter free-flow, except for single mode applications targeting specific polypeptides.

\section{Concluding remarks}

We have developed a prototype multi-channel gel electrophoresis instrument capable of high resolution separation and fast and continuous fraction collection over a broad range of masses. The strategy of using multiple, short linear gels to achieve a separation power similar to that of a long gradient gel and then eluting proteins in a continuous and parallel fashion has been demonstrated effective, particularly for SDS-gels. In a typical 2.5-hour SDS Counter Free-Flow PAGE run, each sample can be separated and eluted into some 
48 to 96 fractions over the mass range of $\sim 10-150 \mathrm{kD}$; sample recovery rates can be $50 \%$ or more; each channel can be loaded with up to $\sim 0.3 \mathrm{mg}$ of protein in $0.4 \mathrm{ml}$; and a purified protein band will elute in $0.4-0.6 \mathrm{ml}$. For native gel electrophoresis, protein aggregation remains a major challenge that limits sample loading capacity to $\sim 50 \mu \mathrm{g}$ per channel and even then does not provide the same resolution as SDS gels. We suspect that native gels could be improved by developing electrophoresis protocols that do not cause significant aggregation and by implementing elution techniques that inhibit band-dilution.

Our current Counter Free-Flow PAGE protocols could be further optimized for specific needs. To improve resolution of low-molecular weight proteins or peptides, one could use higher acrylamide concentrations and/or longer gels, increase the counter-flow rate, and collect smaller fractions. To reduce dilution in the high molecular range, one might use lower acrylamide concentrations in the upper gel segment in the multi mode and increase the electric field. However, there are limits to the optimization of certain variables. For example, separation properties will become less reproducible as acrylamide concentrations approach 3\% or less. Similarly, higher electric fields are more likely to generate air bubbles at the interface between the gel and the conduit, which will change the electrical field, sometimes completely interrupting electrophoresis.

\section{Acknowledgements}

We are grateful to many members of the Protein Complex Analysis Project (PCAP) for their support and for discussions. This work was conducted under Department of Energy contract DE-AC02-05CH11231 awarded to Lawrence Berkeley National Laboratory.

\section{References}

[1] Ornstein, L. Ann. N.Y. Acad. Sci. 1964, 121, 321-349.

[2] Laemmli, U. K. Nature 1970, 227, 680-685.

[3] Jovin, T. M. Ann. N.Y. Sci. 1973, 209, 477-496.

[4] Svensson, H. Acta Chem. Scand. 1961, 15, 325-341.

[5] Vesterberg, O.; Wadstrom, T.; Vesterberg, K.; Svensson, H.; Malmgren, B. Biochim. Biophys. Acta 1967, 133, 435-445.

[6] Camacho-Carvajal, M. M.; Wollscheid, B.; Aebersold, R.; Steimle, V.; Schamel, W. W. Mol. Cell. Proteomics 2004, 3 (2), 176-182.

[7] Butland, G., Peregrin-Alvarez, J. M., Li, J., Yang, W., Yang, X., Canadien, V., Starostine, A.. et al. Nature 2005, 433, 531-537.

[8] Gavin, A. C., Aloy, P., Grandi, P., Krause, R., Boesche, M., Marzioch, M., Rau, C. et al. Nature 2006, 440, 631-636.

[9] Krogan, N. J., Cagney, G., Yu, H., Zhong, G., Guo, X., Ignatchenko, A., Li, J. et al. Nature 2006, 440, 637-643.

[10] Gygi, s., Corthals, G., Zhang, Y., Rochon, Y., Aebersold, R. PNAS 2000, 97 93909395.

[11] Kristjansdottir, K., Wolfgeher, D., Lucius, N., Angulo, D. S., Kron, S. J. Proteome Research 2008, 7, 2812-2824.

[12] Dong, M., Yang, L.,Williams, K., Fisher, S., Hall, S.,Biggin, M.,Jin, J., Witkowska, H. J. Proteome Research, 2008, 7, 1836-1849.

[13] Hjerten, S., Zhu, M.-D. J. Chromatogr. 1985, 327, 157-164. 
[14] Huang, X., Zare, R. N. Anal. Chem. 1990, 62, 443-446.

[15] Muller, O., Foret, F., Karger, B. Anal. Chem. 1995, 67, 2974-2980.

FIGURE LEGENDS:
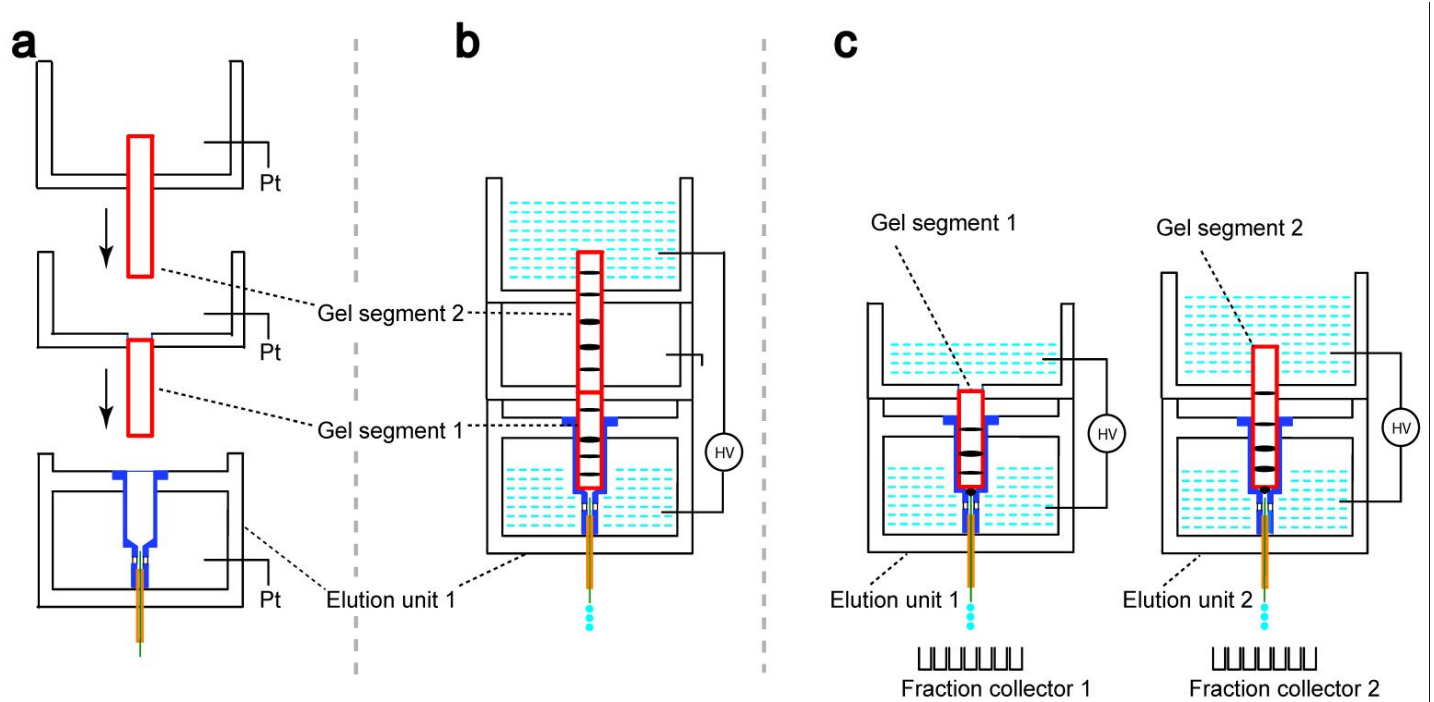

Figure 1. System design and operation scheme for Counter Free-Flow electrophoresis. (a) Shows an elution unit and two separate linear gel columns (Gel segments 1 and 2). Each gel column is attached to a running buffer container that contains a Platinum electrode. The upper column (Gel segment 2) has the lowest polyacrylamide concentration gel and space above the gel for sample loading. The lower column contains a higher polyacrylamide concentration gel. (b) Shows these thee pieces stacked to form an approximation of a gradient gel. Gel segment 1 is inserted into a conduit in elution unit 1, and Gel segment 2 is stacked on top of this. Then, an initial phase of electrophoresis is used to separate proteins from a complex mixture loaded at the top of Gel segment 2. High mobility protein bands migrate into the Gel segment 1 while slow ones remain in Gel segment 2. (c) Illustrates that, after separating (un-stacking) the two gel columns, the buffer container associated with Gel segment 1 is filled, while Gel segment 2 is inserted into a conduit in elution unit 2 (see text and fig-2). Finally, electrophoresis is resumed on both gel columns to further separate and elute the protein bands captured in each gel segment. To stack more than two gel segments in a separation, additional gel boxes designed like Gel segment 1 can be included because they are constructed to allow "self-stacking". 


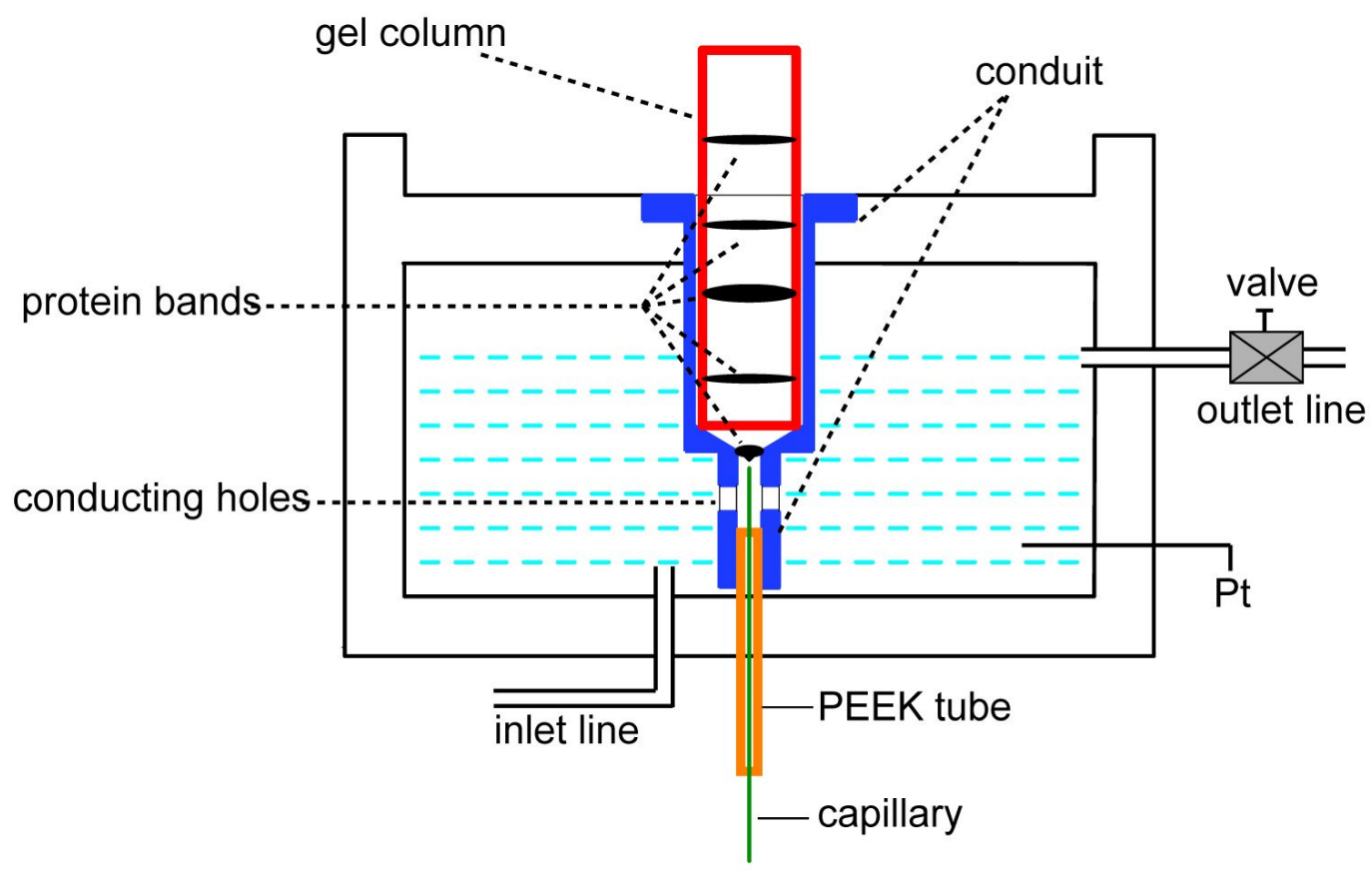

Figure 2. The Counter Free-Flow technique for capturing biomolecules off an electrophoresis gel column. Shown is an elution unit with a single gel column inserted (for clarity the associated upper buffer reservoir is not shown). A capillary tube with a sleeve of PEEK tube is attached to the base plate of the lower (anode) buffer reservoir. Buffer is added to completely fill the reservoir. A conduit of acrylic glass is then inserted from above, fitting over the capillary and PEEK tubes. The tight fit between the PEEK tube and the lower portion of the conduit ensures that the capillary is centered. A gel column consisting of a vertical glass column containing polyacrylamide gel is then inserted down into the conduit as far the taper. Care is taken to avoid trapping air bubbles between the gel column and the taper. After insertion, the buffer level is lowered and maintained at the level indicated by a slow gravity fed inlet and outlet flow so that the electrical contact to the gel is established only through the conducting holes. 


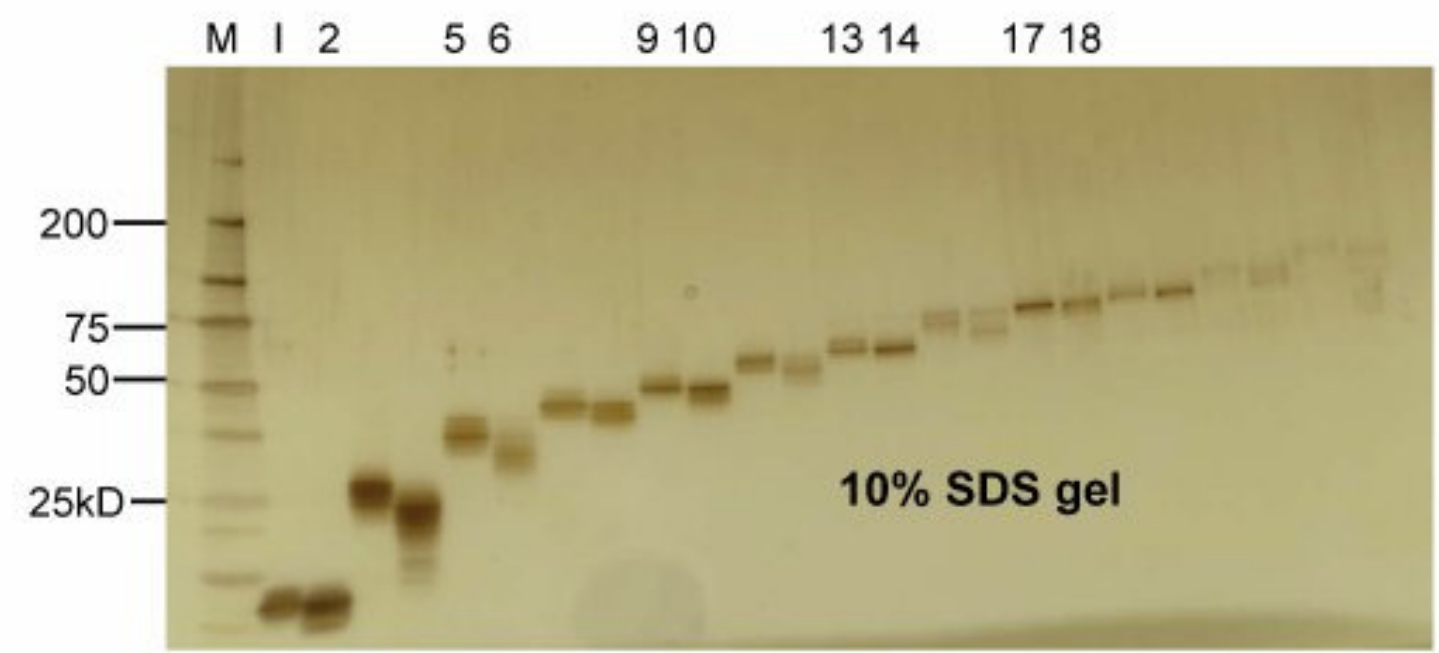

supplementary Fig. 2

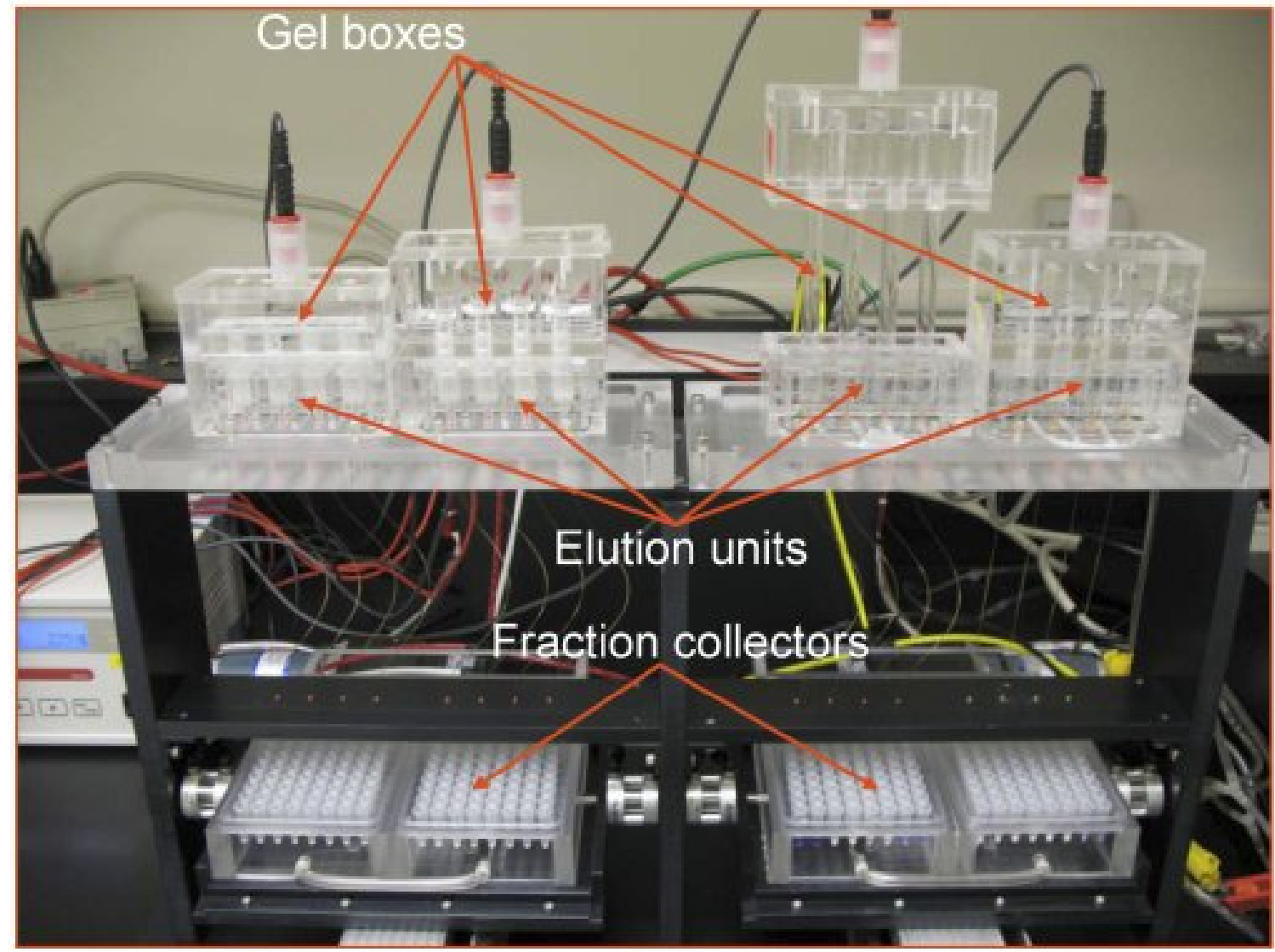

Figure 3. A photograph of the prototype 16-channel Counter Free-Flow electrophoresis and elution apparatus. It includes gel boxes that each house four gel columns of either 3or 5-cm length (top), four elution units (middle) and two motorized fraction collectors (bottom). 
First Stage:

$4.5 \%, 2.5 \mathrm{~cm}$ SDS gel

Second Stage:

$6.5 \%, 5 \mathrm{~cm}$

SDS gel

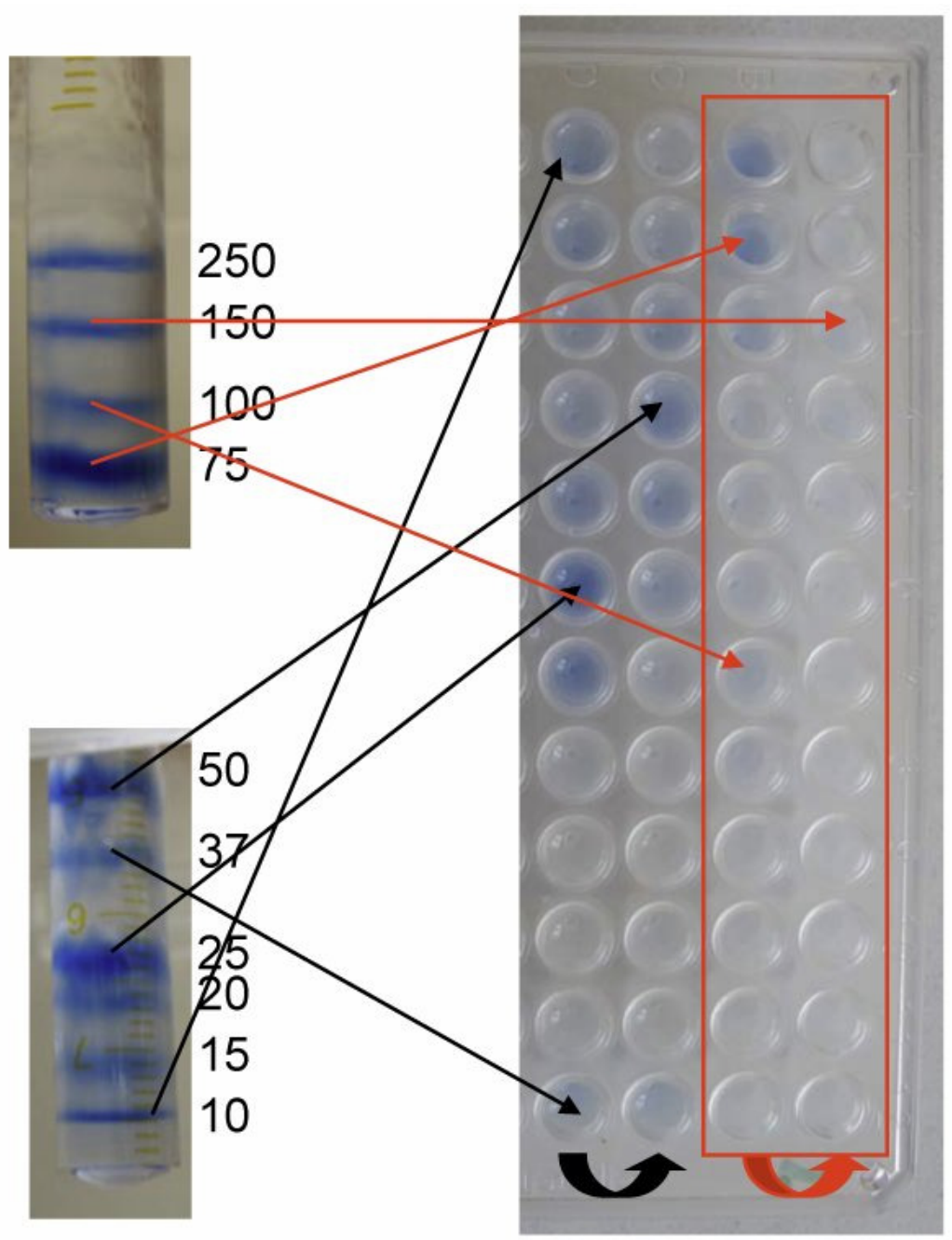

Figure 4. Separating and eluting pre-stained protein markers from a two stage multi mode SDS Counter Free-Flow gel. All 10 pre-stained marker protein bands were clearly separated by two short SDS gels and eluted into $2 \times 24$ fractions. The fact that the four smaller molecular weight polypeptides (10-25 kD) eluted over the first 7 fractions and that the $37 \mathrm{kD}$ band eluted over only two demonstrate the effectiveness of the second stage $6.5 \%$ gel over this size range. The first stage $4.5 \%$ gel was similarly effective for the 75 and $100 \mathrm{kD}$ bands. However, the spread of the $50 \mathrm{kD}$ and $150 \mathrm{kD}$ bands over 5 fractions each also illustrates that sample dilution occurs as protein mobility moves out of the gel's optimum fractionation range. The fractions for each column were collected sequentially from top (left), down, then back up (right) at 2 minutes per fraction. 


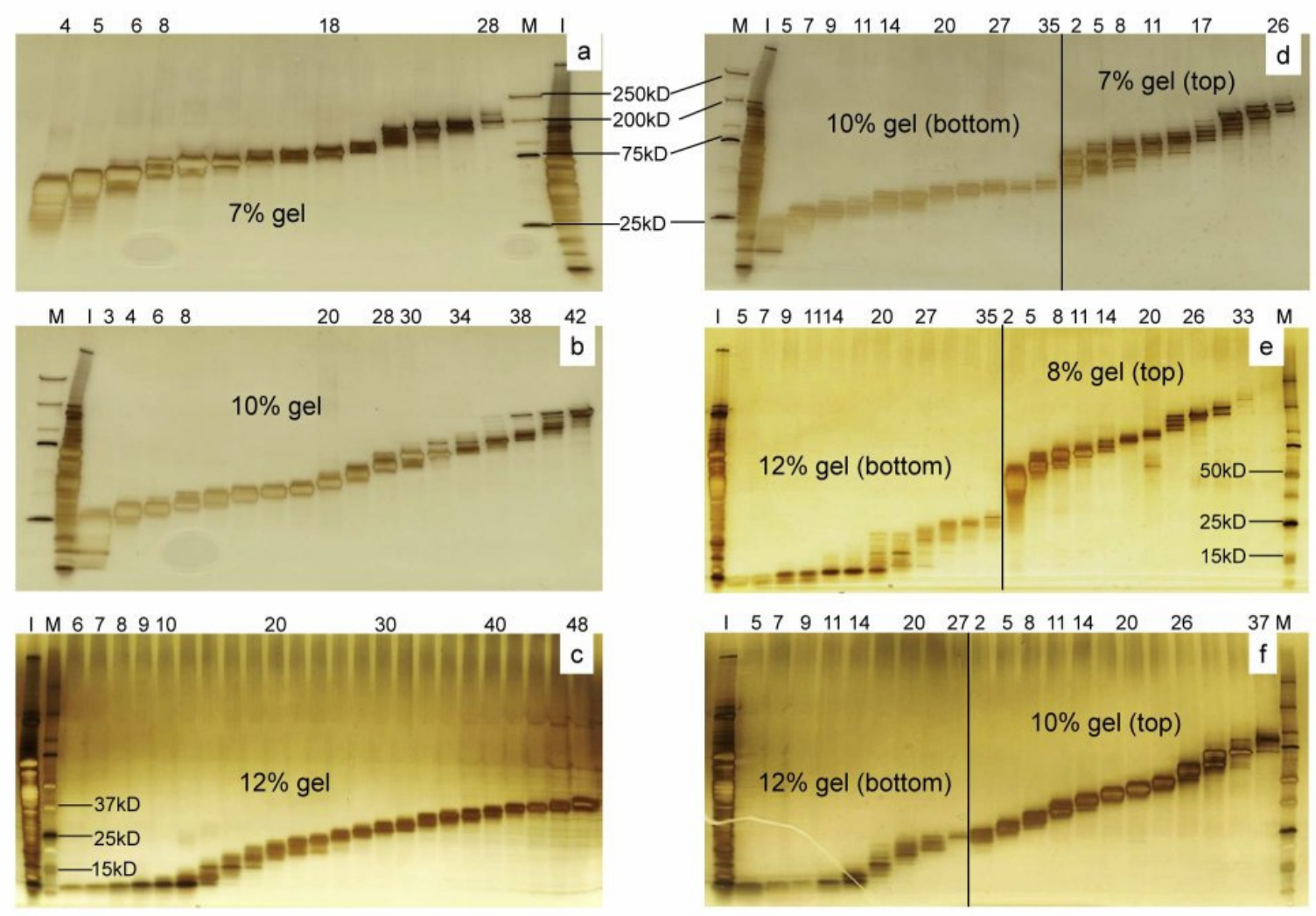

Figure 5. Elution of SDS Counter Free-Flow PAGE gels. Left panels (a, b, c): protein contents of collected fractions of a crude extract of $D$. vulgaris separated using the single mode and different gel concentrations. Right panels (d, e, f): results obtained using twostaged "multi" mode and different gel combinations. The concentrations in the gel columns used are given in each panel. The contents of each eluted fraction were visualized via slab SDS-PAGE and silver staining. The sample input lane is marked as "I," protein markers as "M" and other lanes according to the eluted fraction number. 


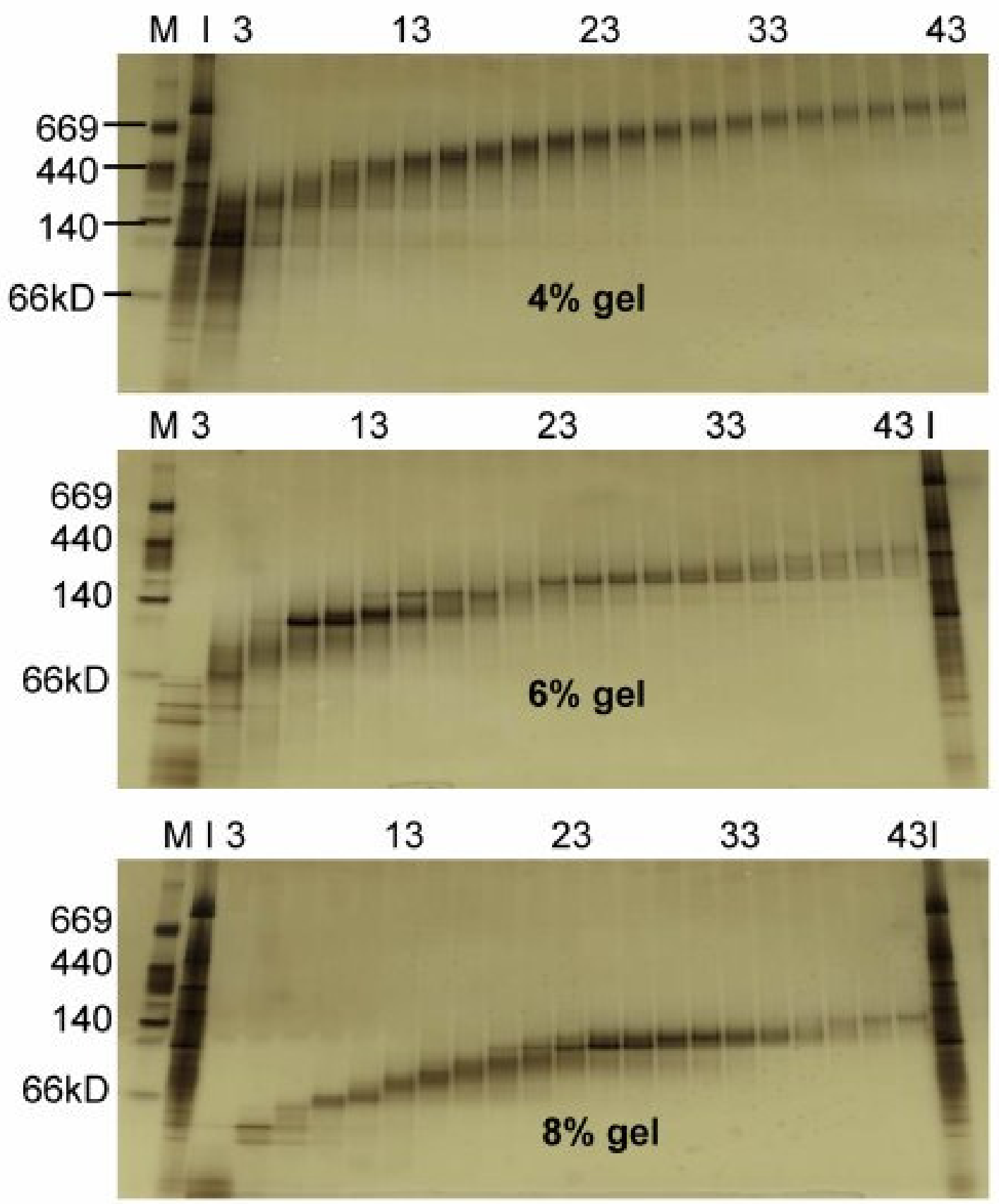

Figure 6. Separation characteristics of linear native gels of 4, 6 and $8 \%$ run in single mode Counter Free-Flow PAGE. The contents of each eluted fraction are visualized via slab native PAGE and silver staining. The lanes are numbered as in Fig. 5. 

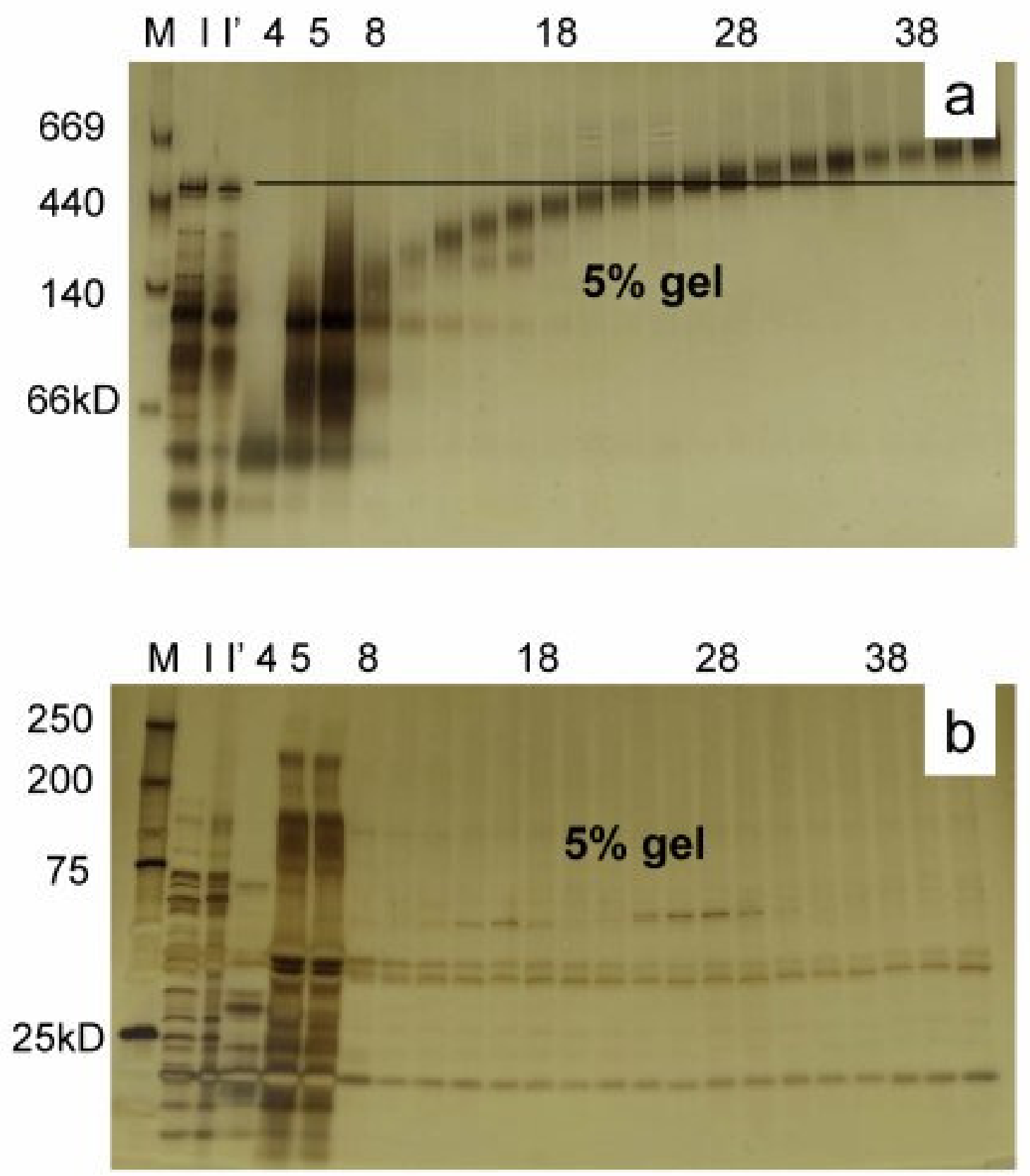

Figure 7. Native Counter Free-Flow PAGE induces protein aggregation. A 5\% gel, single mode Counter Free-Flow electrophoresis of a desalted and concentrated HIC fraction with lower complexity of proteins than a crude extract. Eluted samples were analyzed by (a) native slab gel and (b) SDS slab gel, showing the major protein contents in each fraction. The input samples labeled I and I' are HIC fractions desalted but before and after concentration by a 3-kD column, respectively. 


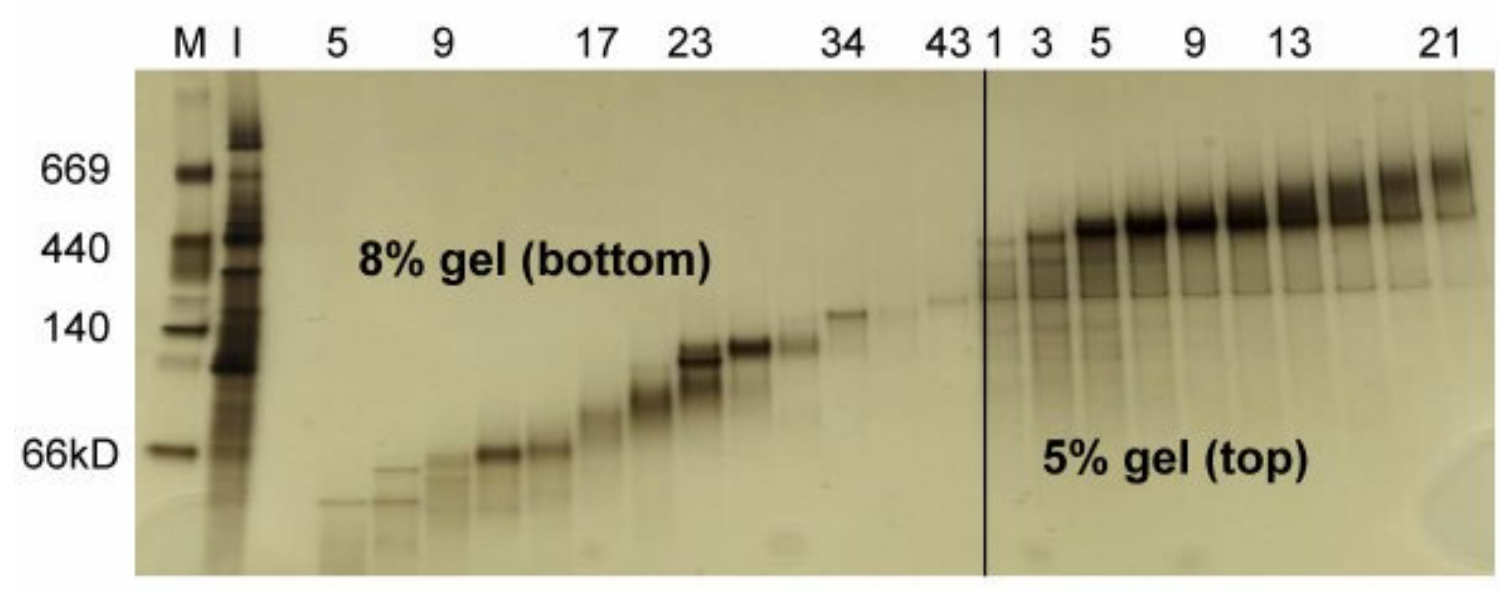

Figure 8. Multi mode run of Native Counter Free-Flow PAGE. Gels of 5\% and $8 \%$ were used to separate a crude extract of $D$. vulgaris in the multi mode. The contents of each eluted fraction are visualized via slab native PAGE and silver staining. 